\title{
Tratamiento superficial de materiales mediante luz solar concentrada: una opción mediante energías renovables
}

\author{
D. Martínez ${ }^{(*)}$ y J. Rodríguez ${ }^{(*)}$
}

\begin{abstract}
Resumen En este artículo se explica de manera ilustrativa la posible aplicación de los hornos solares al tratamiento superficial de materiales. Se realiza una breve descripción de estos sistemas y se da un repaso a las posibles aplicaciones industriales ó de ensayo para las que se ha demostrado su aptitud.

Palabras Clave: Energía solar. Tratamiento superficial. Energías renovables. Temple superficial. Aleación superficial.
\end{abstract}

\section{Materials surface treatments by concentrated solar light: a renewable energy option}

\begin{abstract}
Summary The possible applications of solar furnaces to materials surface treatment are explained in an illustrative manner. A brief description of these systems is exposed, as well as an overview of the feasible industrial or testing applications for which their validity has been proven.
\end{abstract}

Keywords: Solar energy. Surface treatment. Renewable energies. Surface hardening. Surface alloying.

\section{INTRODUCCIÓN}

En este artículo se exponen, desde un enfoque primordialmente ilustrativo, las posibilidades y también las particularidades de los sistemas concentradores de energía solar (hornos solares) con respecto al tratamiento térmico superficial de materiales, planteándose su uso como una posibilidad mas amigable con respecto al medio ambiente frente a otros sistemas más extendidos como láseres o concentradores de arco eléctrico.

La característica diferenciadora de los hornos solares con respecto al resto de sistemas basados en energía solar térmica es que se trata más de un "útil de laboratorio" que de una "herramienta de producción".

En efecto, los Hornos Solares son instalaciones dedicadas a la investigación en los campos de "tra-

(*) CIEMAT-Plataforma Solar de Almería. Apartado 22, 04200-Tabernas. Almería (España) tamiento y caracterización de materiales" y "procesos químicos".

La estructura básica de estas instalaciones consta de un espejo parabólico, y una zona focal de trabajo, generalmente dentro de un edificio, donde se enfoca la luz solar a niveles de concentración muy superiores a los de cualquier otra aplicación solar.

Esta zona de trabajo es muy accesible, basándose generalmente en una mesa ó soporte preparada para la instalación de instrumentación, así como circuitos de refrigeración o cualquier otro útil.

Si a esto se añade un adecuado conocimiento y control de la energía luminosa que se aporta, se tiene entonces la posibilidad de realizar ensayos muy concretos en las mismas condiciones que en un laboratorio convencional.

Por supuesto, esta configuración tan flexible permite realizar fácilmente trabajos preparatorios y simples modificaciones de las configuraciones experimentales, así como realizar investigaciones paralelas, todo ello con gastos moderadamente pequeños. 
También se puede usar como banco de pruebas preliminar para pequeños modelos de receptores para centrales solares con aplicaciones termoeléctricas (Fig. 1).

Los Hornos Solares son especialmente adecuados para ensayos a muy altas temperaturas $(T>$ $1000{ }^{\circ} \mathrm{C}$ ) y también para aquellos en los que el aporte energético sea necesario en forma de "choque térmico". Esto es posible gracias a la altísima concentración de la luz solar, la cual es recogida a unos niveles de densidad que raramente exceden $0,1 \mathrm{~W} / \mathrm{cm}^{2}$, pero que, una vez concentrada puede llegar a alcanzar valores por encima de $1000 \mathrm{~W} / \mathrm{cm}^{2}$ aplicables de manera casi instantánea sobre la muestra a ensayar. Esto permite, en teoría, alcanzar una temperatura de "cuerpo negro" de $3500{ }^{\circ} \mathrm{C}$.

\section{COMPONENTES PRINCIPALES DE UN HORNO SOLAR}

Si seguimos el camino recorrido por los rayos solares, tenemos:

\subsection{Heliostatos}

Son los encargados de hacer el seguimiento solar y redirigir los rayos hacia el disco parabólico. $\mathrm{Al}$ contrario que en las centrales de torre, sus facetas son lo mas planas posible ya que no se trata de concentrar la luz en un blanco, sino de reorientarla.

El grado de planitud de dichas facetas, así como la precisión en el apunte son las características críti-

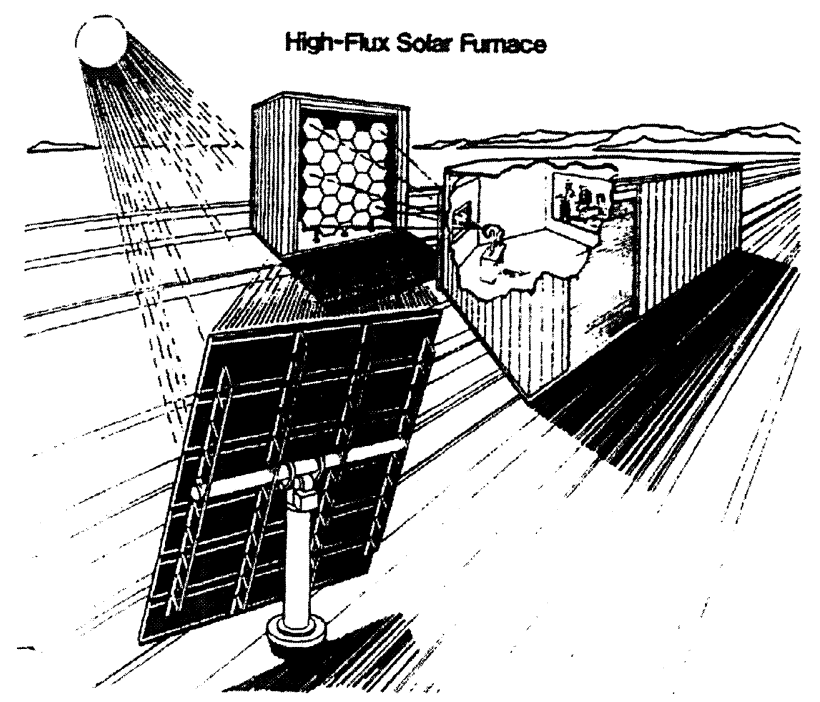

FIG. 1.- Configuración básica de un horno solar (Fuente: NREL) cas del heliostato en cuanto a la calidad del foco que se pretende conseguir.

\subsection{Shutter o Atenuador}

Es el elemento que controla la potencia en el foco. Esto se hace mediante la mayor ó menor apertura del mismo, lo que se suele hacer mediante ordenador.

Puede estar situado entre los heliostatos y el disco, o bien entre el disco y el plano focal. $\mathrm{Su}$ característica crítica es el tiempo de apertura/cierre.

La segunda opción de las mencionadas es muy interesante cuando se pretende realizar ensayos de "choque térmico", ya que un shutter cerca del plano focal es mucho mas pequeño, lo que permite un accionamiento mas enérgico del mismo y por lo tanto tiempos de apertura/cierre menores. Por otro lado, presenta el inconveniente de trabajar en una región espacial donde ya hay concentración, por lo que debe considerarse la necesidad de incorporarle una refrigeración.

\subsection{Concentrador}

Es un disco parabólico faceteado y estático, cuyo eje óptico coincide con la dirección de incidencia de los rayos solares.

Sus características críticas en cuanto a la eficacia en el foco a conseguir son una geometría muy precisa y una buena especularidad.

Para la elaboración de los elementos reflectantes se usan metales reflexivos, polímeros metalizados ó vidrios también metalizados. Para minimizar los errores de especularidad y optimizar los fotones ultravioleta reflejados se tiende a usar espejos con una primera superficie de aluminio depositado por vacío ó por "sputtering", y una segunda capa de un material dieléctrico como puede ser $\mathrm{SiO}_{2}$.

\subsection{Mesa de ensayo}

Es el soporte necesario para situar la pieza en el foco con la mayor precisión posible, así como la aparamenta necesaria (aislamiento, cableado,etc..)

En algunos hornos la mesa de ensayo tiene la capacidad de situarse espacialmente y en mando remoto con precisiones de hasta $1 \mathrm{~mm}$. Asimismo, otra posibilidad es la de realizar secuencias preprogramadas de movimientos dentro de la zona focal; esto puede ser de especial interés cuando se pretenda ensayar piezas de forma compleja o realizar ciclos distintos de los típicos. 


\section{RELACIÓN DE CONCENTRACIÓN Y ERRORES ÓPTICOS}

La estimación del pico de flujo $q_{\text {peak }}$ debido a la geometría de la óptica puede obtenerse de la siguiente ecuación: (1)

$$
q_{\text {peak }}=\rho_{H} \cdot \rho_{C} \cdot I_{\text {max }} \cdot C_{\text {peak }}\left[\mathrm{kW} / \mathrm{m}^{2}\right]
$$

donde el flujo pico depende fundamentalmente de la concentración máxima $C_{\text {peak }}$, la cual viene dada por:

$$
C_{\text {peak }}=\frac{\operatorname{sen}^{2} \Phi}{\operatorname{sen}^{2} \Gamma} \exp \left(-\frac{1}{2} \times\left(\frac{2 \times \sigma_{\text {Tot }}}{\Gamma}\right)^{2}\right)
$$

donde: $\Phi$ es el ángulo de apertura del disco $\Gamma$ es el semiángulo del disco solar $\sigma_{\text {Tot }}$ es el error óptico total del sistema, dado por:

$$
\sigma_{\mathrm{Tot}}=\left(\sigma_{H}^{2}+\sigma_{C}^{2}\right)^{1 / 2}
$$

donde: $\sigma_{H}$ es el error óptico total del heliostato $\sigma_{C}$ es el error óptico total del concentrador

con

$$
\sigma_{H}=\left(\sigma_{H b}^{2}+\left(2 \times \sigma_{H T}\right)^{2}+\sigma_{H M A}^{2}\right)^{1 / 2}
$$

$$
\sigma_{H b}=\left(\left(2 \times \sigma_{H S W}\right)^{2}+\sigma_{H S P}^{2}\right)^{1 / 2}
$$

donde: $\sigma_{H T}$ corresponde al error de seguimiento $\sigma_{H M A}$ corresponde al error de canteo $\sigma_{H S W}$ a la ondulación superficial de los espejos

$\sigma_{H S P}$ al error de especularidad

La relación equivalente para el error óptico total del concentrador $\mathrm{s}_{\mathrm{C}}$ es

con

$$
\sigma_{\mathrm{C}}=\left(\sigma^{2} C b+\sigma^{2} C A\right)^{1 / 2}
$$

$$
\sigma_{C b}=\left(\left(2 \times \sigma_{C S W}\right)^{2}+\left(2 \times \sigma_{C S C}\right)^{2}+\sigma_{C S P}^{2}\right)^{1 / 2}
$$

donde: $\sigma_{C S C}$ corresponde al error de curvatura en la superficie

$\sigma_{C A}$ a la exactitud en el alineamiento

$\sigma_{C S W}$ a la ondulación de la superficie de los espejos

$\sigma_{C S P}$ a la especularidad de las facetas del concentrador

Siendo además: $\rho_{H}$ : Reflectividad de los espejos del Heliostato

$\rho_{C}:$ Reflectividad de los espejos del

Concentrador

$I_{\max }:$ Intensidad de la Radiación

Solar

\section{APLICACIONES DE LOS HORNOS SOLARES}

Comparando brevemente con otros sistemas para el tratamiento superficial de materiales, como los concentradores de arco eléctrico y los láseres, se podría decir lo siguiente:

Los concentradores de arco eléctrico pueden ofrecer concentraciones de 100 a $500 \mathrm{~W} / \mathrm{cm}^{2}$ con densidades de pico (considerando un perfil de Gauss) de hasta $2200 \mathrm{~W} / \mathrm{cm}^{2}$. Por contra presentan dificultades para trabajar con pulsos fotónicos.

En cuanto a los láseres, presentan un amplio abanico de potencias desde 1 hasta $50 \mathrm{KW}$, son monocromáticos y pueden trabajar en modo continuo ó a pulsos. Pueden ofrecer densidades energéticas muy elevadas, del orden de $10^{3}$ hasta $10^{10} \mathrm{~W} / \mathrm{cm}^{2}$.

Por su parte, los hh.ss. existentes actualmente en el mundo, presentan potencias desde $1 \mathrm{KW}$ hasta $5000 \mathrm{KW}$ (considerando los hornos insertados en torre), en cuanto a densidades, valores típicos son 5 $\mathrm{MW} / \mathrm{m}^{2}$ y $20 \mathrm{MW} / \mathrm{m}^{2}$ usando reconcentradores.

Podemos enumerar las principales ventajas de los hornos solares como las siguientes:

a) Se pueden procesar de una vez superficies mucho mas grandes que con los otros procedimientos.

b) Se pueden dar pulsos cortos con rápidas velocidades de calentamiento.

c) Suponen un mínimo impacto ambiental y tiene un alto rendimiento en la conversión energética.

d) $\mathrm{Su}$ espectro es idóneo para materiales que absorben en el visible, evitando el uso de recubrimientos especiales.

Por otro lado, se pueden enumerar como limitaciones las siguientes:

a) Intermitencia del aporte energético

b) La intensidad varía a lo largo del día.

\subsection{Temple y endurecimiento superficial de aceros}

Esta es, por ahora, la aplicación mas extendida, aunque el auge de este tipo de instalaciones (por ejemplo: el 'Paul Scherrer Institut' suizo inauguró un nuevo horno solar en su centro de Villigen durante 1997) augura una diversificación en las actividades.

Esta técnica es muy utilizada en la industria para mejorar las propiedades mecánicas de componentes de máquinas sometidos a altas tensiones ó desgastes; como por ejemplo coronas y rodamientos (Fig. 2). 


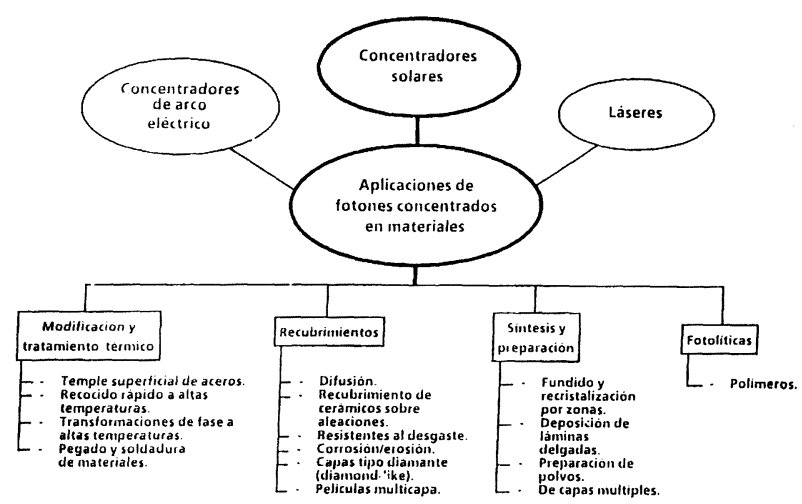

FIG. 2.- Diagrama resumen de aplicaciones de los hornos solares en materiales (Fuente: CIEMAT)

El proceso de endurecimiento local de aceros conlleva un calentamiento local muy intenso, siendo la temperatura de transformación austenítica de unos $720{ }^{\circ} \mathrm{C}$. La fase crítica es el enfriamiento posterior, que debe ser lo mas rápido posible para que la capa superficial se transforme en martensita, que es la fase dura y resistente al desgaste.

Ensayos de temple de diferentes tipos de acero se han venido realizando exitosamente en el Horno Solar de la Plataforma Solar de Almería por grupos de investigadores de varias universidades europeas (Lovaina, Dublin, Londres), así como del "Centro Nacional de Investigaciones Metalúrgicas" perteneciente al C.S.I.C. (2)

Los trabajos se centran ahora en estudiar posibles procedimientos para uniformizar los espesores tratados, así como determinar el valor máximo del área tratable de una vez, en cada ciclo. La determinación de aquellos tipos de acero susceptibles de ser tratados mediante energía solar es otro de los objetos de estudio.

\subsection{Recubrimientos mediante polvos aplicados previamente}

Los recubrimientos se usan como protectores de la corrosión, del desgaste y para la fabricación de superficies roscadas y piezas móviles.

Esta técnica supone un gran ahorro de materiales caros ya que la pieza puede fabricarse en materiales baratos y posteriormente recubrirse con una pequeña aportación de los materiales más costosos.

Las principales dificultades que surgen en estos procesos tienen que ver con el control de la atmósfera durante el ensayo y también con la necesidad de situar el foco sobre un plano horizontal ya que de lo contrario la capa de polvo se desprendería o deslizaría de las probetas. Este requerimiento no plantea dificultades en los hornos de eje óptico vertical, y puede solventarse en los de eje óptico horizontal mediante una placa de aluminio ó cobre, con un pulido de alta calidad para aumentar su reflectividad hasta casi el $100 \%$. Dicha placa se sitúa en el plano focal con una inclinación de $45^{\circ}$ y debidamente refrigerada, con lo que, a costa de una pequeña dispersión del haz, se reorienta hacia un plano horizontal.

Los polvos absorben la radiación muy bien a través de reflexiones múltiples y se calientan rápidamente debido a la escasa conductividad térmica hacia el substrato. Las temperaturas en los ensayos de revestimiento suelen ser de 1000 a $1500{ }^{\circ} \mathrm{C}$, y los tiempos entre 4 y $30 \mathrm{~s}$.

Volviendo a la influencia de la atmósfera en el ensayo, si se produce oxidación del substrato por la presencia de oxígeno y las altas temperaturas, el polvo fundido no llega a mojar la superficie por lo que no se produce el pegado metalúrgico. Esto lleva a la necesidad de emplear atmósfera controlada, siendo imprescindible una cámara de vacío.

Un aspecto a destacar de la utilización de la energía solar para este proceso es que se genera una interfase entre el substrato y el polvo fundido. Esta interfase es una zona de difusión que da "continuidad" a la estructura resultante. Esto no se produce con las técnicas de spray de plasma ó de llama, donde el polvo se adhiere al substrato de manera mecánica.

También hay que decir que el procedimiento solar permite un control de la exposición que conlleva una capacidad de decisión acerca del crecimiento y la orientación del grano.

\subsection{Recubrimientos por CVD (Deposición química en fase vapor)}

Esta posibilidad fue demostrada por investigadores franceses del IMP-Odeillo en 1975. (3)

En este proceso, la sustancia en fase gaseosa reacciona en presencia de un substrato caliente, dando lugar a recubrimientos superficiales. La superficie caliente del substrato puede actuar solamente como fuente calorífica para calentar los reactivos que reaccionarán entre sí para depositarse, ó bien puede existir una reacción gas/substrato para producir un compuesto ó una aleación.

En la cámara de alto vacío del SERI, en Estados Unidos, se ha experimentado satisfactoriamente en la deposición de películas de carbono sobre substrato de $\mathrm{Ni}$ y de $\mathrm{Si}$ usando $\mathrm{CH}_{4}$ y $\mathrm{H}_{2}$ como precursores. (4)

En el caso del Si se han producido películas delgadas de $\mathrm{SiC}$ y también de diamante artificial. Estos films son interesantes cómo materiales electrónicos, ya que proporcionan aislamiento eléctrico parecido al $\mathrm{SiO}_{2}$ y, a la vez, tienen mayor conductividad que el $\mathrm{Cu}$. 
Otra utilidad de estos diamantes artificiales es como recubrimiento de baja fricción. Debido a su extrema dureza, bajo coeficiente de rozamiento y alta conductividad térmica, resultan interesantes para herramientas de corte y superficies de rodamientos.

\subsection{Reacciones gas/sólido para crecimiento de fibras cerámicas}

Además de los ensayos con CVD para deposición de recubrimientos, podemos utilizar la técnica CVD para producir fibras cerámicas.

Las CVD tienen grandes velocidades de deposición, producen recubrimientos más isótropos, más adherentes y son aplicables a la mayoría de óxidos, nitruros, carburos y boruros. Sus principales desventajas son las altas temperaturas necesarias y el manejo de gases corrosivos y explosivos.

Estas reacciones pueden ser de distintos tipos, p.e. reducción, termolisis y desplazamiento.

Son dignos de mención los estudios realizados en el Instituto Tecnológico de Atlanta para producir fibras de HfC. (5)

Hay dos vías para la producción de fibras según las fases en juego; V-S ó V-L-S. En los procesos V$S$ los reactivos gaseosos condensan directamente sobre la fibra sólida. Para el proceso V-L-S se usa un catalizador líquido para nuclear y acelerar la "precipitación" del material solido debajo de la disolución. Las gotas del catalizador permanecen siempre arriba disolviendo nuevos reactivos del gas y trasfiriéndolos a la fibra.

\subsection{Resistencia de materiales}

Las aplicaciones de los hh.ss. no se circunscriben solo a la transformación de materiales especiales, sino que también incluyen el ensayo de materiales entre sus posibles usos.

El hecho de usar hornos solares viene dado por la inexistencia de hornos eléctricos ó antorchas de plasma lo suficientemente grandes y con la flexibilidad necesaria para someter piezas tan grandes a ciclos representativos del calentamiento en la reentrada.
Un ejemplo muy cercano de esto es la realización en la Plataforma Solar de Almería (PSA) de ensayos termomecánicos de diferentes partes de la estructura de protección térmica para el proyecto de transbordador europeo "Hermes".

Estos ensayos se han venido haciendo desde 1987, y las piezas ensayadas han sido distintas "losas" del fuselaje, bordes de ataque, y finalmente el morro del aparato.

Tales componentes han sido fabricados de manera casi artesanal por dos compañías aerospaciales francesas: SEP y AEROSPATIALE, y las especificaciones de ensayo han venido dadas por la compañía DASSAULT AVIATION; todo ello bajo los auspicios de la ESA (Agencia Espacial Europea).

Los ensayos se han realizado en dos hornos de torre, es decir calentados directamente por un campo de heliostatos, en este caso el campo CESA1 de la PSA, en cuya torre se habilitaron dos salas de ensayo para tal fin.

A grandes rasgos, los ensayos han consistido en someter las piezas de $\mathrm{C}-\mathrm{C}, \mathrm{C}-\mathrm{SiC}$, ó $\mathrm{Si}-\mathrm{SiC}$, a ciclos térmicos hasta $1500{ }^{\circ} \mathrm{C}$ con una velocidad de calentamiento de $5{ }^{\circ} \mathrm{C} / \mathrm{s}$, manteniendo los $1500{ }^{\circ} \mathrm{C}$ durante unos minutos para luego bajar a $-2.5^{\circ} \mathrm{C} / \mathrm{s}$ hasta temperatura ambiente.

\section{REFERENCIAS}

(1) Del ARco, J.: "Energetic Levels, Optical Error and Attainable Temperature in the PSA Solar Furnace", PSA Internal Report R-21 bis/91, Noviembre 1991

(2) Rodriguez Donoso, Gloria. Tesis Doctoral 'Aplicaciones de la Energía Solar Concentrada a la Modificación Superficial de Aceros'. CENIM, Madrid, 1994.

(3) Armas, B. et alt.: "Chemical Vapor Deposition of $\mathrm{NbB}_{2}$ and $\mathrm{TaB}_{2}$ through Heating by Concentration of Solar Radiation", Proceedings of Fifth Int. Conf. on CVD, Princeton 1975

(4) PItTS, J.R. et alt.: "Surface Modification Technologies using Concentrated Solar Radiation", Proceedings of Potential Applications of Concentrated Solar Energy, Golden, Noviembre 1990.

(5) LACKEY, W.J.: "Solar-Enhanced CVD Production of Ceramic Whiskers", Proceedings of the 4th. Int. Symp. on Solar Thermal Technology, Santa Fe, Junio 1988. 